\title{
Decomposable graphs and definitions with no quantifier alternation
}

\author{
Oleg Pikhurko ${ }^{1 \dagger}$, Joel Spencer $^{2}$ and Oleg Verbitsky ${ }^{3 \ddagger}$ \\ ${ }^{1}$ Department of Math Sciences, Carnegie Mellon University, Pittsburgh, PA 15213 \\ ${ }^{2}$ Courant Institute, New York University, New York, NY 10012 \\ ${ }^{3}$ Institut für Informatik, Humboldt Universität Berlin, Berlin, D-10099
}

Let $D(G)$ be the minimum quantifier depth of a first order sentence $\Phi$ that defines a graph $G$ up to isomorphism in terms of the adjacency and the equality relations. Let $D_{0}(G)$ be a variant of $D(G)$ where we do not allow quantifier alternations in $\Phi$. Using large graphs decomposable in complement-connected components by a short sequence of serial and parallel decompositions, we show examples of $G$ on $n$ vertices with $D_{0}(G) \leq 2 \log ^{*} n+O(1)$. On the other hand, we prove a lower bound $D_{0}(G) \geq \log ^{*} n-\log ^{*} \log ^{*} n-O(1)$ for all $G$. Here $\log ^{*} n$ is equal to the minimum number of iterations of the binary logarithm needed to bring $n$ below 1 .

Keywords: descriptive complexity of graphs, first order logic, Ehrenfeucht game on graphs, graph decompositions

\section{Introduction}

Given a finite graph $G$, how succinctly can we describe it using first order logic and the laconic language consisting of the adjacency and the equality relations? A first order sentence $\Phi$ defines $G$ if $\Phi$ is true precisely on graphs isomorphic to $G$. All natural succinctness measures of $\Phi$ are of interest: the length $L(\Phi)$ (a standard encoding of $\Phi$ is supposed), the quantifier depth $D(\Phi)$ which is the maximum number of nested quantifiers in $\Phi$, and the width $W(\Phi)$ which is the number of variables used in $\Phi$ (different occurrences of the same variable are not counted). All the three characteristics inherently arise in the analysis of the computational problem of checking if a $\Phi$ is true on a given graph [3]. They give us a small hierarchy of descriptive complexity measures for graphs: $L(G)$ (resp. $D(G), W(G)$ ) is the minimum $L(\Phi)$ (resp. $D(\Phi), W(\Phi)$ ) of a $\Phi$ defining $G$. These graph invariants will be referred to as the logical length, depth, and width of $G$. We have $W(G) \leq D(G) \leq L(G)$. The former number is of relevance for graph isomorphism testing, see [2]. $W(G)$ and $D(G)$ admit a purely combinatorial characterization in terms of the Ehrenfeucht game, see [2,8].

We here address the logical depth of a graph. We focus on the following general question: How do restrictions on logic affect the descriptive complexity of a graph? Call a first order sentence $\Phi$ to be

\footnotetext{
$\dagger$ Partially supported by NSF Grant DMS 0457512.

¥Supported by an Alexander von Humboldt fellowship. 
a-alternation if it contains negations only in front of relation symbols and every sequence of nested quantifiers in $\Phi$ has at most $a$ quantifier alternations. Let $D_{a}(G)$ denote a variant of $D(G)$ for $a$-alternation defining sentences, so $D(G) \leq D_{a+1}(G) \leq D_{a}(G)$. The logic of 0 -alternation sentences is most restrictive and provably weaker than the unbounded first order logic. Whereas the problem of deciding if a first order sentence is satisfiable by some graph is unsolvable, it becomes solvable if restricted to 0-alternation sentences (the latter due to Ramsey's logical work [7] founding the combinatorial Ramsey theory).

It is not hard to observe that $D_{0}(G) \leq n+1$ where $n$ denotes the number of vertices in $G$. This bound is in general best possible as $D\left(K_{n}\right)=n+1$. Nevertheless, it admits a non-obvious improvement under a rather small restriction on the automorphism group of $G$. If the latter does not contain any transposition of two vertices, then $D_{1}(G) \leq(n+5) / 2$, see [6]. No sublinear improvement is possible because of the sequence of asymmetric graphs with $W(G)=\Omega(n)$ constructed in [2]. In [4] we prove that $D(G)=\log _{2} n-\Theta\left(\log _{2} \log _{2} n\right)$ and $D_{0}(G) \leq(2+o(1)) \log _{2} n$ for almost all $G$.

After obtaining these worst-case and average-case results, we undertake a "best-case" analysis in [5]. We define the succinctness function $q(n)=\min \{D(G): G$ has order $n\}$ and show that its values may be superrecursively small if compared to $n: f(q(n)) \geq n$ for no recursive $f$. A weaker but still surprising succinctness result is also obtained for the fragment of first order logic with no quantifier alternation. Let $q_{0}(n)=\min \left\{D_{0}(G): G\right.$ has order $\left.n\right\}$.

Theorem $1 q_{0}(n) \leq 2 \log ^{*} n+O(1)$ for infinitely many $n$.

In [5] this theorem is proved by considering $G$ in a certain class of asymmetric trees and estimating $D_{0}(G)$ in terms of the radius of a tree. We here reprove this result by showing the same definability phenomenon in a different class of graphs. We consider $G$ in a class of graphs with small complementconnected induced subgraphs and estimate $D_{0}(G)$ in terms of the number of the serial and parallel decompositions [1] decomposing $G$ in the complement-connected components.

We also present a new result complementing Theorem 1.

Theorem $2 q_{0}(n) \geq \log ^{*} n-\log ^{*} \log ^{*} n-O(1)$ for all $n$.

As a consequence, $q_{0}(n) \leq f(q(n))$ for no recursive $f$, which also shows a superrecursive gap between the graph invariants $D(G)$ and $D_{0}(G)$.

\section{Definitions}

We use the following notation: $V(G)$ is the vertex set of a graph $G$; $\operatorname{diam} G$ is the diameter of $G$; $\bar{G}$ is the complement of $G ; G \sqcup H$ is the disjoint union of graphs $G$ and $H ; G \subset H$ means that $G$ is isomorphic to an induced subgraph of $H$ (we will say that $G$ is embeddable in $H$ ); $G \sqsubset H$ means that $G$ is isomorphic to the union of some of the connected components of $H$.

We call $G$ complement-connected if both $G$ and $\bar{G}$ are connected. An inclusion-maximal complementconnected induced subgraph of $G$ will be called a complement-connected component of $G$ or, for brevity, cocomponent of $G$. Cocomponents have no common vertices and partition $V(G)$.

The decomposition of $G$, denoted by $D e c G$, is the set of all connected components of $G$ (this is a set of graphs, not just isomorphism types). Furthermore, given $i \geq 0$, we define the depth $i$ decomposition of $G$ by $D e c_{0} G=\operatorname{Dec} G$ and $D e c_{i+1} G=\bigcup_{F \in D e c_{i} G} D e c \bar{F}$. Note that $P_{i}=\left\{V(F): F \in D e c_{i} G\right\}$ is a partition of $V(G)$ and that $P_{i+1}$ refines $P_{i}$. The depth $i$ environment of a vertex $v \in V(G)$, denoted by $\operatorname{Env}_{i}(v)$, is the $F$ in $\operatorname{Dec}_{i} G$ containing $v$. 
We define the rank of a graph $G$, denoted by $r k G$, inductively as follows: (1) If $G$ is complementconnected, then $r k G=0$. (2) If $G$ is connected but not complement-connected, then $r k G=r k \bar{G}$. (3) If $G$ is disconnected, then $r k G=1+\max \{r k F: F \in D e c G\}$. In other terms, $r k G$ is the smallest $k$ such that $P_{k+1}=P_{k}$ or such that $P_{k}$ consists of $V(F)$ for all cocomponents $F$ of $G$.

In the Ehrenfeucht game on two disjoint graphs $G$ and $H$ two players, Spoiler and Duplicator, alternatingly select vertices of the graphs, one vertex per move. Spoiler starts and is always free to move in any of $G$ and $H$; Then Duplicator must choose a vertex in the other graph. Let $x_{i} \in V(G)$ and $y_{i} \in V(H)$ denote the vertices selected by the players in the $i$-th round. Duplicator wins the $k$-round game if the component-wise correspondence between $x_{1}, \ldots, x_{k}$ and $y_{1}, \ldots, y_{k}$ is a partial isomorphism from $G$ to $H$; Otherwise the winner is Spoiler. In the O-alternation game Spoiler plays all the game in the same graph he selects in the first round.

Assume $G ¥ H$. Let $D(G, H)$ (resp. $D_{0}(G, H)$ ) denote the minimum $D(\Phi)$ over (resp. 0-alternation) first order sentences $\Phi$ that are true on one of the graphs and false on the other. The Ehrenfeucht theorem relates $D(G, H)$ and the length of the Ehrenfeucht game on $G$ and $H$. We will use the following version of the theorem: $D_{0}(G, H)$ is equal to the minimum $k$ such that Spoiler has a winning strategy in the $k$-round 0 -alternation Ehrenfeucht game on $G$ and $H$. It is also useful to know that $D_{0}(G)=\max \left\{D_{0}(G, H): H \neq G\right\}$.

We define the tower-function by Tower $(0)=1$ and $\operatorname{Tower}(i)=2^{\operatorname{Tower}(i-1)}$ for each subsequent $i$.

\section{Upper bound: Proof of Theorem 1}

Lemma 1 Consider the Ehrenfeucht game on graphs $G$ and $H$. Let $x, x^{\prime} \in V(G), y, y^{\prime} \in V(H)$ and assume that the pairs $x, y$ and $x^{\prime}, y^{\prime}$ are selected by the players in the same rounds. Furthermore, assume that $\operatorname{Env}_{l}(x) \neq \operatorname{Env}_{l}\left(x^{\prime}\right), \operatorname{Env}_{l}(y)=\operatorname{Env}_{l}\left(y^{\prime}\right)$, and diam Env $v_{i}(y) \leq 2$ for every $i \leq l$. Then Spoiler can win in at most $l+1$ rounds (l rounds if $G$ is connected), playing all the time in $H$.

Proof: We proceed by induction on $l$. The base case is $l=0$ if $G$ is disconnected and $l=1$ if $G$ is connected. If $y$ and $y^{\prime}$ are adjacent in $\operatorname{Env}_{l}(y)$, Duplicator has already lost. Otherwise, Spoiler uses the fact that $\operatorname{diam}_{\operatorname{Env}}(y)=2$ and selects $y^{\prime \prime}$ adjacent in $\operatorname{Env}_{l}(y)$ to both $y$ and $y^{\prime}$. Duplicator cannot do so with any $x^{\prime \prime}$ because $x$ and $x^{\prime}$ are in different components of $G$ if $l=0$ or $\bar{G}$ if $l=1$.

Assume that $l \geq 1$. Let $0 \leq m \leq l$ be the minimum number such that $x^{\prime} \notin \operatorname{Env}_{m}(x)$. If $m<l$, Spoiler wins in the next $m+1 \leq l$ moves by induction. If $m=l$, Spoiler uses the same trick as in the base case and forces Duplicator to make a move $x^{\prime \prime}$ outside $E n v_{l-1}(x)$. By the induction hypothesis, Spoiler needs $l$ extra moves to win.

As long as Duplicator avoids meeting the conditions of Lemma 1 (in particular, selects $x^{\prime} \in \operatorname{Env}_{l}(x)$ whenever Spoiler selects $y^{\prime} \in \operatorname{Env}_{l}(y)$ ), we will say that she bewares of the environment threat.

Let $r k G=k$. We call $G$ uniform if $D e c_{k-1} G$ contains no complement-connected graph, that is, every cocomponent appears in $D e c_{k} G$ and no earlier. We call $G$ inclusion-free if the following two conditions are true for every $i<k$ : (1) For any $K \in D e c_{i} G, \bar{K}$ contains no isomorphic connected components. (2) If two elements $K$ and $M$ of $D e c_{i} G$ are non-isomorphic, then neither $\bar{K} \sqsubset \bar{M}$ nor $\bar{M} \sqsubset \bar{K}$.

Lemma 2 (Main Lemma) Let $G$ be a uniform inclusion-free graph. Suppose that every cocomponent of $G$ has exactly c vertices. Then $D_{0}(G) \leq 2 r k G+c+1$. 
Proof: Let $r k G=k$. Fix a graph $H \neq F G$. We will design a strategy allowing Spoiler to win the 0alternation Ehrenfeucht game on $G$ and $H$ in at most $2 k+c+1$ moves. Since $D_{0}(G)=D_{0}(\bar{G})$, without loss of generality we will assume that $G$ is connected. Since the case of $k=0$ is trivial, we will also assume that $k \geq 1$.

Case 1: $H$ has a cocomponent $C$ non-embeddable in any cocomponent of $G$. If $C$ has no more than $c$ vertices, Spoiler selects all $C$. Otherwise he selects $c+1$ vertices spanning a complement-connected subgraph in $C$ (it is not hard to show that this is always possible). If Duplicator's response $A$ is within a cocomponent of $G$, then $C \not A$ by the assumption. Otherwise $A$ is not complement-connected and Duplicator loses anyway.

In the sequel we will assume that Duplicator bewares of the environment threat during all game.

Case 2: $G \subset H$ or there are $l \leq k$ and $A \in D e c_{l} G$ properly embeddable in some $B \in D e c_{l} H$, and not Case 1. Spoiler plays in $H$. If $G \subset H$, set $A=G, B=H$, and $l=0$. Let $H_{0}$ be a copy of $A$ in $B$. At the first move Spoiler selects an arbitrary $y_{0} \in V(B) \backslash V\left(H_{0}\right)$. Denote Duplicator's response in $G$ by $x_{0}$ and set $G_{0}=E n v_{l}\left(x_{0}\right)$. From now on Spoiler plays in $H_{0}$. Since we are not in Case $1, B$ is not a cocomponent of $H$ and hence $\operatorname{diam} B \leq 2$. Since Duplicator is supposed to beware of the environment threat, from now on she is forced to play in $G_{0}$.

Subcase 2.1: $G_{0} \neq H_{0}$. Assume that $l<k$ (the case of $l=k$ will be covered by the last phase of the strategy). Since $G_{0}$ and $H_{0}$ are non-isomorphic copies of elements of $D e c_{l} G$ and $G$ is inclusion-free, Spoiler is able to make his next choice $y_{1}$ in some $H_{1} \in \operatorname{Dec} \overline{H_{0}}$ absent in $\operatorname{Dec} \overline{G_{0}}$. Denote Duplicator's response in $G_{0}$ by $x_{1}$ and set $G_{1}=E n v_{l+1}\left(x_{1}\right)$. Note that $G_{1}$ and $H_{1}$ are non-isomorphic copies of elements of $D e c_{l+1} G$. Playing in the same fashion in the subsequent $k-l-1$ rounds, Spoiler finally achieves the players' moves in some non-isomorphic $G_{k-l} \in D e c_{k} G$ and $H_{k-l}$, the latter being a copy of an element of $D e c_{k} G$. Both the graphs have $c$ vertices. Now Spoiler selects the $c-1$ remaining vertices of $H_{k-l}$ and wins whatever Duplicator's response is.

Subcase 2.2: $G_{0} \cong H_{0}$. Though the graphs are isomorphic, the crucial fact is that $G_{0}$, unlike $H_{0}$, contains a selected vertex. By the definition of an inclusion-free graph, every automorphism of $A \cong$ $G_{0} \cong H_{0}$ takes each cocomponent onto itself. Therefore every isomorphism between $G_{0}$ and $H_{0}$ matches cocomponents of these graphs in the same way. Let $Y$ be the counterpart of $E n v_{k}\left(x_{0}\right)$ in $H_{0}$ with respect to this matching. In the second round Spoiler selects an arbitrary $y_{1}$ in $Y$. Denote Duplicator's answer by $x_{1}$. If $x_{1} \in \operatorname{Env}_{k}\left(x_{0}\right)$, Spoiler selects all $Y$ and wins. Otherwise there is $m \leq r k A$ such that $\operatorname{Env}_{m}\left(x_{1}\right)$ in $G_{0}$ and $\operatorname{Env}_{m}\left(y_{1}\right)$ in $H_{0}$ are non-isomorphic. This allows Spoiler to apply the strategy of Subcase 2.1.

Case 3: Neither Case 1 nor Case 2. Spoiler plays in $G_{0}=G$. His first move $x_{0}$ is arbitrary. Denote Duplicator's response in $H$ by $y_{0}$ and set $H_{0}=E n v_{0}\left(y_{0}\right)$. Since we are not in Case 2, $G_{0} \not \subset H_{0}$. As $G_{0}$ is inclusion-free, $\overline{G_{0}}$ has a connected component $G_{1}$ with no isomorphic copy in $\overline{H_{0}}$. Spoiler selects $x_{1}$ arbitrarily in $G_{1}$. Let Duplicator respond with $y_{1}$ somewhere in $H_{0}$ and denote $H_{1}=\operatorname{Env}_{1}\left(y_{1}\right)$. Thus $G_{1} \neq H_{1}$ and $G_{1} \not \subset H_{1}$, the latter again because we are not in Case 2. In the next round Spoiler again selects a vertex in a component $G_{2}$ of $\overline{G_{1}}$ absent in $\overline{H_{1}}$. Continuing in the same fashion, Spoiler finally forces playing the game on some $G_{m} \in D e c_{m} G_{0}$ and $H_{m} \in D e c_{m} H_{0}$ with $G_{m} \not \subset H_{m}$ under one of the two terminal conditions: (1) $m<k$ and $H_{m}$ (or its complement) is a cocomponent of $H$. (2) $m=k$. In the first case note that, as we are not in Case $1, H_{m}$ is embeddable in some cocomponent of $G$ (or its complement) and hence has at most $c$ vertices. Therefore it suffices for Spoiler to select altogether $c+1$ vertices in $G_{m}$ to win (recall the assumption that Duplicator bewares of the environment threat and hence cannot move outside $H_{m}$ ). In the second case $G_{m}$ is a cocomponent of $G$ and hence has $c$ vertices. Spoiler selects all $G_{m}$. Since Duplicator's response must be complement-connected, she is forced to play 
within a cocomponent of $H_{m}$ and hence loses.

Length of the game. The above strategy allows Spoiler to win in at most $k+c$ moves under the condition that Duplicator bewares of the environment threat. If Duplicator ignores this threat, Spoiler needs $k+1$ additional moves according to Lemma 1.

Let $R_{0}$ consist of all complement-connected graphs of order 5. Assume that $R_{i-1}$ is already specified. Fix $F_{i}$ to be the family of all $\left\lfloor\left|R_{i-1}\right| / 2\right\rfloor$-element subsets of $R_{i-1}$. Define $R_{i}$ to be the set of the complements of $\bigsqcup_{G \in S} G$ for all $S$ in $F_{i}$. Note that $R_{i}$ consists of inclusion-free uniform graphs of rank $i$ whose cocomponents all have 5 vertices. All graphs in $R_{i}$ have the same order; Denote it by $N_{i}$. Let $M_{i}=\left|R_{i}\right|$. By the construction,

$$
M_{i+1}=\left(\begin{array}{c}
M_{i} \\
\left\lfloor M_{i} / 2\right\rfloor
\end{array}\right)=\sqrt{\frac{2+o(1)}{\pi M_{i}}} 2^{M_{i}} \text { and } N_{i+1}=\left\lfloor M_{i} / 2\right\rfloor N_{i}>M_{i} .
$$

A simple estimation shows that $N_{i} \geq \operatorname{Tower}(i-O(1))$. To complete the proof of Theorem 1 , choose $G_{i}$ in $R_{i}$. Using Main Lemma, we obtain $q_{0}\left(N_{i}\right) \leq D_{0}\left(G_{i}\right) \leq 2 i+6 \leq 2 \log ^{*} N_{i}+O(1)$.

\section{Lower bound: Proof-sketch of Theorem 2}

Let $L_{a}(G)$ denote the minimum length of an $a$-alternation sentence defining $G$.

Lemma $3 L_{a}(G) \leq$ Tower $\left(D_{a}(G)+\log ^{*} D_{a}(G)+O(1)\right)$.

An analog of this lemma for $L(G)$ and $D(G)$ appears in [5] but its proof does not work under restrictions on the alternation number. The proof of Lemma 3 will appear in the full version.

Given $n$, denote $k=q_{0}(n)$ and fix a graph $G$ on $n$ vertices such that $D_{0}(G)=k$. By Lemma 3, $G$ is definable by a 0 -alternation $\Phi$ of length at most $\operatorname{Tower}\left(k+\log ^{*} k+O(1)\right)$. Using the standard reduction, we convert $\Phi$ to an equivalent prenex $\exists^{*} \forall^{*}$-sentence $\Psi$ (i.e. existential quantifiers in $\Psi$ all precede universal quantifiers). Since the reduction does not increase the total number of quantifiers, $D(\Psi) \leq L(\Phi)$. It is well known and easy to prove that, if a prenex $\exists^{*} \forall^{*}$-sentence $\Psi$ is true on some structure, then it is true on some structure of order at most $D(\Psi)$. Since the $\Psi$ is true only on $G$, we have $n \leq D(\Psi) \leq L(\Phi) \leq$ Tower $\left(k+\log ^{*} k+O(1)\right)$, which proves the theorem.

\section{References}

[1] A. Brandstädt, V. B. Le, J. P. Spinrad. Graph classes: a survey. SIAM Monographs (1999).

[2] J.-Y. Cai, M. Fürer, N. Immerman. An optimal lower bound on the number of variables for graph identification. Combinatorica 12:389-410 (1992).

[3] E. Grädel. Finite model theory and descriptive complexity. In: Finite Model Theory and Its Applications. Texts in Theoretical Computer Science, an EATCS Series. Springer (2005).

[4] J. H. Kim, O. Pikhurko, J. Spencer, O. Verbitsky. How complex are random graphs in first order logic? Random Structures and Algorithms 26:119-145 (2005).

[5] O. Pikhurko, J. Spencer, O. Verbitsky. Succinct definitions in the first order theory of graphs. Accepted for publication in Annals of Pure and Applied Logic. (arxiv.org/abs/math. LO/0401307) 
[6] O. Pikhurko, H. Veith, O. Verbitsky. First order definability of graphs: tight bounds on quantifier rank. Submitted (2004) (arxiv.org/abs/math.co/0311041)

[7] F. Ramsey. On a problem of formal logic. Proc. London Math. Soc. 2-nd series 30:264-286 (1930).

[8] J. Spencer. The strange logic of random graphs. Springer Verlag (2001). 